\title{
A QUASI-ANOSOV DIFFEOMORPHISM THAT IS NOT ANOSOV $\left({ }^{1}\right)$
}

\author{
BY \\ JOHN FRANKS AND CLARK ROBINSON
}

\begin{abstract}
In this note, we give an example of a diffeomorphism $f$ on a three dimensional manifold $M$ such that $f$ has a property called quasi-Anosov but such that $f$ does not have a hyperbolic structure (is not Anosov). Mañé has given a method of extending $f$ to a diffeomorphism $g$ on a larger dimensional manifold $V$ such that $g$ has a hyperbolic structure on $M$ as a subset $V$. This gives a counterexample to a question of $M$. Hirsch.
\end{abstract}

M. Hirsch asks in [2], if a diffeomorphism $g: V \rightarrow V$ has a compact invariant submanifold $M \subset V$ with a hyperbolic structure as a subset of $V$, does it folfollow that $g$ restricted to $M$ is Anosov (has a hyperbolic structure). He proves this is true in certain cases if $g$ has a dense orbit in $V$. Ricardo Mañe notes that $g$ restricted to $M$ has a property he calls quasi-Anosov [5]. He asks if a quasiAnosov diffeomorphism is always Anosov. C. Robinson [6] gives an example of a quasi-Anosov flow (not a diffeomorphism) that is not Anosov on an eleven dimensional manifold. In this note, we give an example of a quasi-Anosov diffeomorphism $f$ on a three dimensional manifold. (This is the minimal dimension.) Mañé gives a method in [5] of embedding our result in a diffeomorphism $g$ of a manifold $V$ such that $g$ has a hyperbolic structure on $M$. This gives a counterexample to the question of Hirsch as stated above. However, we do not know if $g$ can be constructed so $M$ is contained in the nonwandering set of $g$. Also, the results of Hirsch [2] show that if our $f: M^{3} \rightarrow M^{3}$ is embedded in $g: V \rightarrow V$ so that $g$ has a hyperbolic structure on $M$ and the dimension of $V$ is four or five, then $g$ cannot have a point with a dense orbit in all of $V$.

1. Definitions and Theorem. A diffeomorphism is quasi-Anosov if the fact that $\left|T f^{n} v\right|$ is bounded for all $n \in Z$ implies that $v=0$. Here $T f$ is the induced map on tangent vectors of $M, v \in T M$. If $\Lambda \subset M$ is invariant by a diffeomorphism $f$, we say that $f$ has a hyperbolic structure on $\Lambda$ if there are $0<\lambda<1, C>0$, and a splitting $T M \mid \Lambda=E^{u} \oplus E^{s}$ such that for $n \geqslant 0$,

Received by the editors June 30, 1975.

AMS (MOS) subject classifications (1970). Primary 58F15.

(1) Research was partially supported by the National Science Foundation, GP42329X. 


$$
\begin{gathered}
\left|T f^{n} v^{s}\right| \leqslant C \lambda^{n}\left|v^{s}\right| \text { for } v^{s} \in E^{s}, \\
\left|T f^{-n} v^{u}\right| \leqslant C \lambda^{n}\left|v^{u}\right| \text { for } v^{u} \in E^{u} .
\end{gathered}
$$

A diffeomorphism $f$ on $M$ is Anosov if $f$ has a hyperbolic structure on $M$. The nonwandering set, $\Omega$, of $f$ is the set of points $x \in M$ such that for each neighborhood $U$ of $x$ there is an integer $n>0$ such that $f^{n}(U) \cap U \neq \varnothing$.

We define the stable and unstable manifolds at all points $x \in M$ by $W^{s}(x)=$ $\left\{y \in M: d\left(f^{n} x, f^{n} y\right) \rightarrow 0\right.$ as $\left.n \rightarrow \infty\right\}$ and $W^{u}(x)=\left\{y \in M: d\left(f^{n} x, f^{n} y\right) \rightarrow 0\right.$ as $n \rightarrow-\infty\}$. If $f$ has a hyperbolic structure on the nonwandering set then these are actually manifolds [3]. If we also assume the periodic points are dense in $\Omega$, then by [4] or [10] each point $y$ of $M$ is on the stable and unstable manifolds of some point in $\Omega$. Also $\left\{v \in T_{x} M:\left|T f^{n} v\right|\right.$ is bounded for $\left.n \leqslant 0\right\}=T_{x}\left[W^{u}(x)\right]$ and $\left\{v \in T_{x} M:\left|T f^{n} v\right|\right.$ is bounded for $\left.n \geqslant 0\right\}=T_{x}\left[W^{s}(x)\right]$. See [3]. Therefore under these conditions if $T_{x}\left[W^{u}(x)\right] \cap T_{x}\left[W^{s}(x)\right]=\left\{0_{x}\right\}$ for all $x$ in $M$, then $f$ is quasi-Anosov. See [4] or [10] for more definitions and basic facts of the theory.

THEOREM. Let $M$ be the connected sum of two copies of the three torus. There is a diffeomorphism $f$ on $M$ that is quasi-Anosov but not Anosov.

Remarks. 1. The diffeomorphism $f$ has two hyperbolic invariant subsets and the dimension of the stable bundle on the source is two and on the sink is one. Since the dimension of $E^{s}$ is not constant $f$ cannot be Anosov. However $T_{x}\left[W^{u}(x)\right] \cap T_{x}\left[W^{s}(x)\right]=\left\{0_{x}\right\}$ for all $x$ in $M$, so $f$ is quasi-Anosov.

2. A quasi-Anosov diffeomorphism on a two dimensional manifold is Anosov, so our example is in the lowest possible dimension. To prove this statement, note that if $f$ is quasi-Anosov on a two manifold then $f$ has a hyperbolic structure on $\Omega$ as approved in [5], [7], or [9]. Since $f$ is quasi-Anosov, all the splitting must be one dimensional (exercise). Since the splittings have constant dimensions on $\Omega$ [2], [5], and [7] all prove that $f$ has a hyperbolic splitting on all of $M$. Therefore $f$ is Anosov.

2. Proof of Theorem. We first construct a " $D A$ " diffeomorphism on $M_{1}$ $=T^{3}$ with certain linearity properties near the source.

LEMMA 1. Let $M_{1}=T^{3}$; there exists a diffeomorphism $f_{1}: M_{1} \rightarrow M_{1}$ which leaves invariant a one dimensional foliation $F$ and has the following properties:

(1) There is a point $p \in M_{1}$ which is a source for $f_{1}$ and a neighborhood $U$ of $p$ with local coordinates $x_{1}, x_{2}, x_{3}$ which are $C^{\infty}$ except at $p$ and such that 


$$
U=\left\{q \in M_{1} \mid\left(\sum_{i=1}^{3} x_{i}(q)^{2}\right)^{1 / 2}<9\right\} .
$$

(2) If $q, f_{1}(q) \in U$ then $x_{i}\left(f_{1}(q)\right)=2 x_{i}(q)$; i.e., in the $x_{i}$ coordinates $f_{1}$ is multiplication by 2 .

(3) The leaves of the foliation $F$ restricted to $U$ are given by $x_{2}(q)=$ constant, $x_{3}(q)=$ constant.

(4) If $\Lambda_{1}=\bigcap_{n>0} f_{1}^{n}\left(M_{1}-U\right)$, then $\Lambda_{1}$ is a compact invariant hyperbolic set whose stable manifolds are the leaves of the foliation $F$ restricted to $M_{1}-\{p\}$.

Proof. The diffeomorphism $f_{1}$ is a " $D A$ " on $M_{1}=T^{3}$. This is a wellknown construction in dimension 2 [10] or [11]; we include an exposition of the dimension $3 D A$ construction as an appendix for completeness. However for the moment we need only that $f_{1}$ is a perturbation of a hyperbolic toral automorphism which had a two dimensional unstable foliation and one dimensional stable foliation. The perturbation changes a hyperbolic fixed point to a source, but preserves the stable foliation so it remains invariant under $f_{1}$. If $p$ is the source and $V$ is any sufficiently small neighborhood of $p$, then $\Lambda_{1}=\bigcap_{n>0} f_{1}^{n}\left(M_{1}-V\right)$ has a hyperbolic structure and its stable manifolds are the leaves of $F$ (the stable foliation of the hyperbolic toral automorphism) restricted to $M_{1}-\{p\}$ (see Appendix for proof).

We assume the original hyperbolic toral automorphism $g$ was based on a matrix which has distinct real eigenvalues, one between 0 and 1 and the other two greater than 1. For example,

$$
\left(\begin{array}{ccc}
0 & 1 & 0 \\
0 & 0 & 1 \\
1 & -6 & 5
\end{array}\right)
$$

We choose coordinates $u_{1}, u_{2}, u_{3}$ on a neighborhood of $p$ in directions parallel to the eigenspaces of $g$ and such that $p=(0,0,0)$ in these coordinates. We assume (see Appendix) that $f_{1}$ is constructed to be linear on a neighborhood of $p$ in these coordinates, so that the $u_{1}$ direction, the contracting direction of $g$, is an eigendirection for $f_{1}$ with eigenvalue 2 , and so that $f_{1}=g$ on the unstable manifold $W=\left\{q \mid u_{1}(q)=0\right\}$.

Let $w_{1}, w_{2}$ be standard coordinates on $R^{2}$ and define $L: R^{2} \rightarrow R^{2}$ by $L\left(w_{1}, w_{2}\right)=\left(2 w_{1}, 2 w_{2}\right)$. Since any two expanding linear maps of $R^{2}$ are locally conjugate by a homeomorphism which is a $C^{\infty}$ diffeomorphism except at the fixed points, we know that if $D=\left\{w \in R^{2}|| w \mid<9\right\}$ there exists $\varphi: D \rightarrow W$ which is $C^{\infty}$ except at 0 and has $C^{\infty}$ inverse except at $p$ and satisfies $\varphi(L(w))=$ 
$f_{1}(\varphi(w))$ when both sides are defined. Now if $U=\varphi(D)$ and we define coordinates by $x_{1}=u_{1}, x_{2}(q)=w_{1}\left(\varphi^{-1}(q)\right), x_{3}(q)=w_{2}\left(\varphi^{-1}(q)\right)$ then one checks easily that $x_{i}\left(f_{1}(q)\right)=2 x_{i}(q)$. Also the coordinates $x_{1}, x_{2}, x_{3}$ are $C^{\infty}$ except at $p$. Since the curves $x_{2}(q)=$ const, $x_{3}(q)=$ const are the same as the curves $u_{2}(q)=$ const, $u_{3}(q)=$ const, these are the leaves of the foliation $F$ restricted to $U$. This proves the lemma.

Next, we use $f_{1}: M_{1} \rightarrow M_{1}$ constructed in the lemma to construct a preliminary diffeomorphism on the connected sum of two copies of $T^{3}$. Let $F$ be the stable foliation of $f_{1}$ (one dimensional). Let $f_{2}: M_{2} \rightarrow M_{2}$ be another copy of the same thing, with one dimensional foliation $F^{\prime}$. Let $V$ be an open set on $M_{2}$ and $y_{1}, y_{2}, y_{3}$ local coordinates analogous to $U$ and $x_{1}, x_{2}, x_{3}$ on $M_{1}$, but such that $F^{\prime}$ is given by $y_{1}(q)=$ const, $y_{2}(q)=$ const. Define ||$_{1}$ on $U$ and ||$_{2}$ on $V$ by $|q|_{1}^{2}=\sum_{i=1}^{3} x_{i}(q)^{2}$ and $\left|q^{\prime}\right|_{2}^{2}=\Sigma_{i=1}^{3} y_{i}\left(q^{\prime}\right)^{2}$. Let $D_{i}=$ $\left\{\left.z \in U|| z\right|_{i}<1 / 8\right\}$. We will attach $M_{1}-D_{1}$ and $M_{2}-D_{2}$ along a collar neighborhood of these boundaries to form a new manifold $M$ diffeomorphic to the connected sum of two copies of $T^{3}$. Let $A_{1}=\left\{\left.z \in U|1 / 8<| z\right|_{1} \leqslant 8\right\}$ and $A_{2}=$ $\left\{\left.z \in V|1 / 8 \leqslant| z\right|_{2} \leqslant 8\right\}$. We define an attaching diffeomorphism $g: A_{2} \rightarrow A_{1}$ by $g\left(y_{1}, y_{2}, y_{3}\right)=\left(\Sigma y_{i}^{2}\right)^{-1}\left(y_{1}, y_{2}, y_{3}\right)$ in $x_{i}$ coordinates. Thus $g$ sends the circle of radius $r$ in $A_{2}$ to the circle of radius $1 / r$ in $A_{1}$, so the outer boundary of $A_{2}$ is taken to the inner boundary of $A_{1}$ and vice versa. Note also that $g \circ$ $f_{2}^{-1}(z)=f_{1} \circ g(z)$.

We will say that $z_{1} \sim z_{2}$ if $z_{1}=g\left(z_{2}\right)$ and define $M$ to be $\left(M_{1}-D_{1}\right) \cup$ $\left(M_{2}-D_{2}\right) / \sim$. Then $M$ is a $C^{\infty}$ manifold and we define a diffeomorphism $f_{0}$ : $M \rightarrow M$ by $f_{0}(z)=f_{1}(z)$ if $z \in M_{1}-D_{1}$ and $f_{0}(z)=f_{2}^{-1}(z)$ if $z \in M_{2}-D_{2}$. Notice if $z \in\left(M_{1}-D_{1}\right) \cap\left(M_{2}-D_{2}\right)=A_{1} \cup A_{2} / \sim$ and if $z$ is the equivalence class of $q \in A_{2}$ and $q^{\prime} \in A_{1}$, then $g \circ f_{2}^{-1}(q)=f_{1}(g(q))=f_{1}\left(q^{\prime}\right)$ so $f_{2}^{-1}(q) \sim$ $f_{1}\left(q^{\prime}\right)$, and hence $f_{0}$ is well defined.

We will consider the annulus $A=A_{1} \cup A_{2} / \sim$ and use the coordinates $x_{1}$, $x_{2}, x_{3}$ which come from $A_{1}$. Then if $|z|^{2}=|z|_{1}^{2}=\Sigma x_{i}(z)^{2}$, we have $A=$ $\{z|1 / 8 \leqslant| z \mid \leqslant 8\}$.

There are two one dimensional foliations on $A$, the restrictions of $F$ on $M_{1}$ and $F^{\prime}$ on $M_{2}$. We will denote these also by $F$ and $F^{\prime}$. The foliation $F$ consists of straight lines in the $x_{i}$ coordinates but the foliation $F^{\prime}$ is a more complicated "dipole" foliation in these coordinates which will be discussed later. Since there are tangencies of $F$ and $F^{\prime}$, we want to modify $f_{0}$ and $F^{\prime}$ to eliminate these tangencies.

Lemma 2. There exists a $C^{\infty}$ isotopy $h_{t}$ of $A$ such that:

(1) $h_{0}=$ id: $A \rightarrow A$.

(2) $h_{t}(z)=z$ for all $t$ and all $z$ in a neighborhood of the boundary of $A$. 
(3) If $B=\{z \in A|1 / 4 \leqslant| z \mid \leqslant 4\}$ then the foliations $F$ and $h_{1}\left(F^{\prime}\right)$ are nowhere tangent on $B$.

(4) If $z, f_{0}(z)$ are in $B$ then $h_{t}\left(f_{0}(z)\right)=f_{0}\left(h_{t}(z)\right)$, for $0<t<1$.

(5) $\left|h_{t}(z)\right|=|z|$ for $z \in A, 0 \leqslant t \leqslant 1$.

We defer the proof for the moment and show that the existence of this isotopy allows us to finish the construction of a quasi-Anosov diffeomorphism.

Proof OF THEOREM. Define a diffeomorphism $f: M \rightarrow M$ by $f(z)=$ $f_{0}(z)$ if $z \notin A$ and if $z \in A$ by

$$
f(z)= \begin{cases}f_{0}(z), & |z|>1 / 2, \\ h_{1} \circ f_{0} \circ h_{1}^{-1}(z), & |z|<2 .\end{cases}
$$

Here $h_{1}$ is extended to be the identity off of $A$. Notice by (4) of Lemma 2 that $h_{1} \circ f_{0}=f_{0} \circ h_{1}$ on $B$ so $f_{0}(z)=h_{1} \circ f_{0} \circ h_{1}^{-1}(z)$ if $1 / 2<|z|<2$. Thus $f$ is a well-defined diffeomorphism. We must check that it is quasi-Anosov. If $\Lambda_{1}$ and $\Lambda_{2}$ are the compact invariant hyperbolic sets in $M_{1}$ and $M_{2}$ (thought of now as subsets of $M$ ), then $v \in T M_{z}$ and $z \in \Lambda_{i}$ implies $\sup _{n \in Z}\left|D f^{n}(v)\right|=\infty$ because of the hyperbolicity. On the other hand if $z \notin \Lambda_{i}$ then the orbit of $z$ under $f$ intersects the set $C=\{w \in A|1 \leqslant| z \mid \leqslant 2\}$ so it suffices to check tangent vectors at points of this set.

We notice that the parts of the stable manifolds of $\Lambda_{1}$ which lie in $C$ are precisely the leaves of the foliation $F$ restricted to $C$. This is because $\left.f\right|_{M_{1}-D_{1}}=$ $f_{1} l_{M_{1}-D_{1}}$. Hence if $v \in T M_{z}$ and $z \in C$ then if $v$ is not tangent to $F$ it follows that

$$
\lim _{n \rightarrow \infty}\left|D f^{n}(v)\right|=\lim _{n \rightarrow \infty}\left|D f_{1}^{n}(v)\right|=\infty .
$$

Also the parts of the unstable manifolds of $\Lambda_{2}$ which lie in $C$ are the leaves of the foliation $h_{1}\left(F^{\prime}\right)$. This is because $\left.f^{-1}\right|_{M_{2}-D_{2}}=h_{1} \circ f_{2} \circ h_{1}^{-1}$ restricted to $M_{2}-D_{2}$ and the stable manifolds of $f_{2}$ are the leaves of $F^{\prime}$, so since $h_{1}$ conjugates $f_{2}$ and $f^{-1}$ it carries the stable manifolds of $f_{2}$ to those of $f^{-1}$. Thus if $v \in T M_{z}, z \in C$ and $v$ is not tangent to $h_{1}\left(F^{\prime}\right)$, it follows that $\lim _{n \rightarrow \infty}\left|D f^{-n}(v)\right|$ $=\infty$. Any tangent vector at a point of $C$ is not tangent to at least one of the foliations $F$ and $h_{1}\left(F^{\prime}\right)$; hence the diffeomorphism $f: M \rightarrow M$ is quasi-Anosov as desired. We note that $f$ is not Anosov because the two compact hyperbolic sets $\Lambda_{1}$ and $\Lambda_{2}$ have hyperbolic splittings of different dimensions and hence are not the restrictions of a single hyperbolic splitting on all of $M$.

Proof of Lemma 2. First we give a geometric description, and then we write down the equations and verify carefully that there are no tangencies of $F$ 
and $h_{1}\left(F^{\prime}\right)$. In the $x_{i}$ coordinates, $\left(x_{1}(f(q)), x_{2}(f(q)), x_{3}(f(q))\right)=$ $2\left(x_{1}(q), x_{2}(q), x_{3}(q)\right)$ and the foliation $F$ is given by $x_{2}(q)=$ constant, $x_{3}(q)=$ constant, i.e. $F$ is tangent to $i=\partial / \partial x_{1}$. We take "spherical" coordinates $\rho, \varphi, \theta$, on $A$ with $\varphi$ varying between $-\pi / 2$ and $\pi / 2$ instead of 0 to $\pi$ as in usual coordinates. Note that $\varphi=0$ is the equator and $\rho(q)=|q|_{1}$. The attaching map $g$ in local coordinates reflects about the sphere of radius one. Therefore the vertical foliation $F^{\prime}$ goes into the "dipole field".

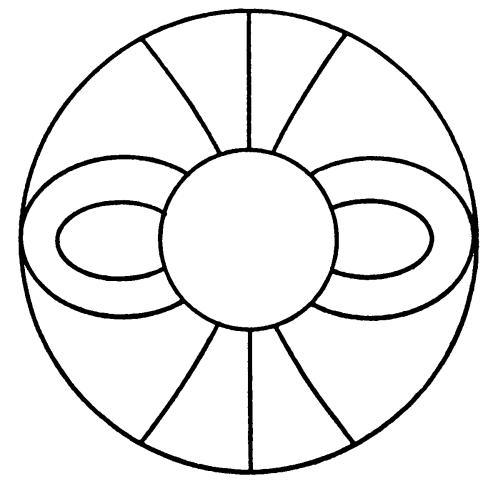

$x_{2}=0$

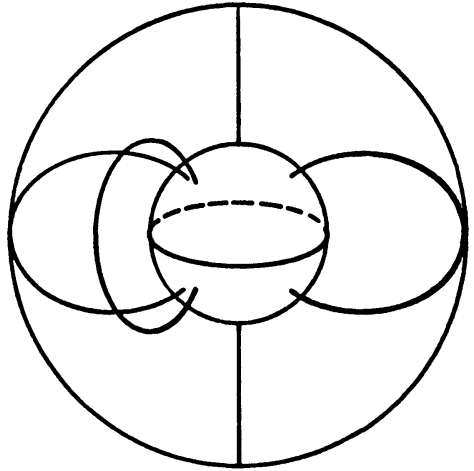

$\ln R^{3}$ In $R^{2}$

We construct the isotopy $h_{t}$ on $B=\left\{\left.q|1 / 4<| q\right|_{1}=\rho(q)<4\right\}$. It preserves $\rho$ and the $\varphi$ and $\theta$ variables are independent of $\rho$. It is then easy to extend it to an isotopy of $A=\{q: 1 / 8 \leqslant \rho(q) \leqslant 8\}$ that is the identity near the boundary.

The first step of the isotopy is to twist the south pole relative to the north pole. The foliation $F^{\prime}$ then twists around the equator. If we orient $F^{\prime}$, we get a vector field that points up near the poles $\varphi= \pm \pi / 2$, and is nearly horizontal near the equator.

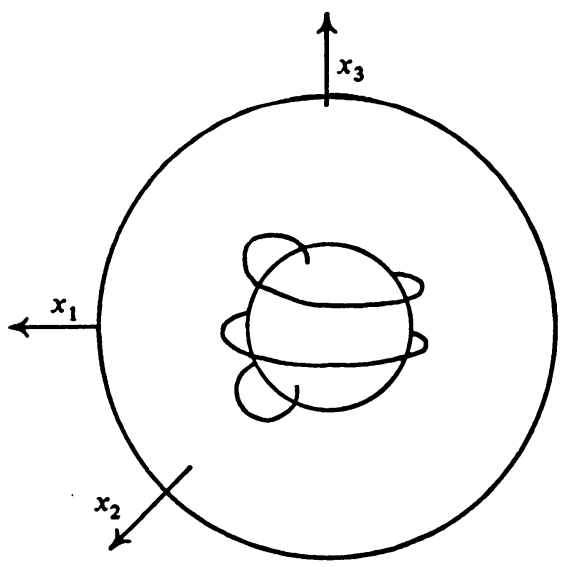


In fact, the map which assigns to a point in $B$ the unit tangent vector field has an image that only covers slightly more than the upper hemisphere of $S^{2}$. The greater the twist, the more horizontal the vector field is at the equator, and the more nearly the image comes to just the upper hemisphere. (The unit tangents to the original $F^{\prime}$ cover $S^{2}$ because they point down at the equator.) The foliation $F$ is tangent to $\underline{i}$. To eliminate tangencies we need to make the unit tangents of $h_{1}\left(F^{\prime}\right)$ miss both $\pm \underline{i}$ (a point and its antipital point). So far the vector field points nearly in the $\underline{i}$ direction near $\varphi=0$ and $\theta= \pm \pi / 2$. We next tilt the foliation up (in the $\underline{k}=\partial / \partial x_{3}$ direction) at these points and let it point down again near $\theta=0, \pi$ where it has some component in the $\underline{j}=\partial / \partial x_{2}$ direction.

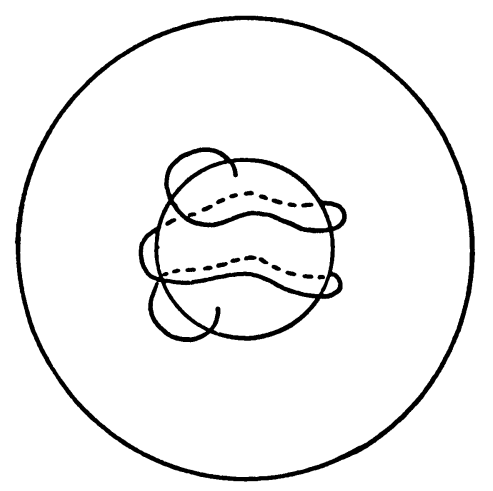

In this way, $h_{1}\left(F^{\prime}\right)$ never points in the $\pm \underline{i}$ direction.

Next we write down the equations and verify completely that $h_{1}\left(F^{\prime}\right)$ is never tangent to $i$. The equations for the attaching map $g$ in spherical coordinates are $\rho(g(q))=\left(\Sigma y_{i}^{2}\right)^{-1 / 2}, \varphi(g(q))=\varphi(q)=\tan ^{-1}\left(y_{3} /\left(y_{1}^{2}+y_{2}^{2}\right)^{1 / 2}\right)$, $\theta(g(q))=\theta(q)=\tan ^{-1}\left(y_{2} / y_{1}\right)$. Therefore the tangent to $F^{\prime}$ is

$$
\frac{\partial \rho}{\partial y_{3}} \frac{\partial}{\partial \rho}+\frac{\partial \varphi}{\partial y_{3}} \frac{\partial}{\partial \varphi}+\frac{\partial \theta}{\partial y_{3}} \frac{\partial}{\partial \theta} .
$$

Then

$$
\begin{aligned}
& \frac{\partial \rho}{\partial y_{3}}=-y_{3}\left(\sum y_{i}^{2}\right)^{-3 / 2}=-\left(\sum x_{i}^{2}\right) y_{3}\left(\sum y_{i}^{2}\right)^{-1 / 2}=-\rho^{2} \sin \varphi, \\
& \frac{\partial \varphi}{\partial y_{3}}=\left(y_{1}^{2}+y_{2}^{2}\right)^{1 / 2}\left(y_{1}^{2}+y_{2}^{2}+y_{3}^{2}\right)^{-1} \\
& =\left(x_{1}^{2}+x_{2}^{2}+x_{3}^{2}\right)^{1 / 2}\left(y_{1}^{2}+y_{2}^{2}+y_{3}^{2}\right)^{-1 / 2} \\
& =\rho \cos \varphi \text {, and } \\
& \frac{\partial \theta}{\partial y_{3}}=0 \text {. }
\end{aligned}
$$


Factoring out $-\rho$ we get a tangent vector field to $F^{\prime}$ to be $\rho \sin \varphi \partial / \partial \rho-$ $\cos \varphi \partial / \partial \varphi$. Let $\beta=\tan ^{-1} 3$ and $\alpha=\tan ^{-1} 2$. Let $\gamma(\varphi)$ be negative, equal to zero in a neighborhood of $\pm \pi / 2$, and constant between $\pm \beta$. We specify its size later. Let $\Gamma(\varphi)=\int_{-\pi / 2}^{\varphi} \gamma(\varphi) d \varphi$.

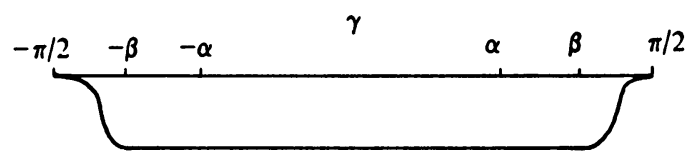

The diffeomorphism

$$
(\rho, \varphi, \theta) \rightarrow\left(\rho^{\prime}, \varphi^{\prime}, \theta^{\prime}\right)=(\rho, \varphi, \theta+\Gamma(\varphi))
$$

adds the twist near the equator. Let $\Delta(\varphi)$ be a positive function that is equal to zero for $|\varphi| \geqslant \beta$ and constant for $\varphi$ between $\pm \alpha$. Let $\delta(\varphi)=\Delta^{\prime}(\varphi)$.
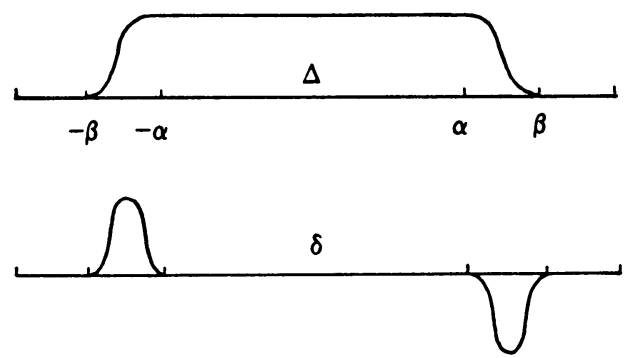

Let $\mu\left(\theta^{\prime}\right)=-1 / 2 \sin 2 \theta^{\prime}$ so $\mu^{\prime}\left(\theta^{\prime}\right)=-\cos 2 \theta^{\prime}$. The diffeomorphism

$$
\left(\rho^{\prime}, \varphi^{\prime}, \theta^{\prime}\right) \rightarrow\left(\rho^{\prime \prime}, \varphi^{\prime \prime}, \theta^{\prime \prime}\right)=\left(\rho^{\prime}, \varphi^{\prime}+\Delta\left(\varphi^{\prime}\right) \mu\left(\theta^{\prime}\right), \theta^{\prime}\right)
$$

pushes up near $\theta^{\prime}= \pm \pi / 2$ (as we shall see). The derivatives of the two diffeomorphisms have matrices

$$
\left(\begin{array}{ccc}
1 & 0 & 0 \\
0 & 1 & 0 \\
0 & \gamma(\varphi) & 1
\end{array}\right) \text { and }\left(\begin{array}{ccc}
1 & 0 & 0 \\
0 & 1+\delta\left(\varphi^{\prime}\right) \mu\left(\theta^{\prime}\right) & \Delta\left(\varphi^{\prime}\right) \mu^{\prime}\left(\theta^{\prime}\right) \\
0 & 0 & 1
\end{array}\right)
$$

Notice if $\left|\delta\left(\varphi^{\prime}\right) \mu\left(\theta^{\prime}\right)\right| \leqslant 1 / 2$ then both matrices are invertible. Since both maps are linear near the poles they are differentiable. All immersions of $S^{2}$ to itself are diffeomorphisms, so these are both diffeomorphisms (isotopic to the identity). Let $h_{1}$ be the composition. The tangent vector to $h_{1}\left(F^{\prime}\right)$ is

$$
\begin{aligned}
X= & \rho \sin \varphi \frac{\partial}{\partial \rho}-\cos \varphi\left[1+\delta(\varphi) \mu\left(\theta^{\prime \prime}\right)+\gamma(\varphi) \Delta(\varphi) \mu^{\prime}\left(\theta^{\prime \prime}\right)\right] \frac{\partial}{\partial \varphi} \\
& -\gamma(\varphi) \cos \varphi \frac{\partial}{\partial \theta},
\end{aligned}
$$




$$
\begin{aligned}
\left(\begin{array}{ccc}
1 & 0 & 0 \\
1 & 1+\delta \mu & \Delta \mu^{\prime} \\
0 & 0 & 1
\end{array}\right) & \left(\begin{array}{lll}
1 & 0 & 0 \\
0 & 1 & 0 \\
0 & \gamma & 1
\end{array}\right)\left(\begin{array}{c}
\rho \sin \varphi \\
-\cos \varphi \\
0
\end{array}\right) \\
& =\left(\begin{array}{c}
\rho \sin \varphi \\
-\cos \varphi\left(1+\delta \mu+\gamma \Delta \mu^{\prime}\right) \\
-\gamma \cos \varphi
\end{array}\right) .
\end{aligned}
$$

Notice $\theta^{\prime \prime}=\theta^{\prime}$ and $\varphi^{\prime}=\varphi$. We want to find the $\underline{j}$ and $\underline{k}$ components of $X$. We compose with the derivative into rectangular coordinates. Remember $-\pi / 2 \leqslant \varphi$ $\leqslant \pi / 2$ so $x_{1}=\rho^{\prime \prime} \cos \theta^{\prime \prime} \cos \varphi^{\prime \prime}, x_{2}=\rho \sin \theta^{\prime \prime} \cos \varphi^{\prime \prime}, x_{3}=\rho^{\prime \prime} \sin \varphi^{\prime \prime}$, and the matrix of the derivative is

$$
\left(\begin{array}{ccc}
\cos \theta^{\prime \prime} \cos \varphi^{\prime \prime} & -\rho \cos \theta^{\prime \prime} \sin \varphi^{\prime \prime} & -\rho \sin \theta^{\prime \prime} \cos \varphi^{\prime \prime} \\
\sin \theta^{\prime \prime} \cos \varphi^{\prime \prime} & -\rho \sin \theta^{\prime \prime} \sin \varphi^{\prime \prime} & \rho \cos \theta^{\prime \prime} \cos \varphi^{\prime \prime} \\
\sin \varphi^{\prime \prime} & \rho \cos \varphi^{\prime \prime} & 0
\end{array}\right) .
$$

The $\underline{j}$ and $\underline{k}$ components are

$$
\begin{aligned}
\rho^{-1} X \cdot \underline{j}= & \sin \theta^{\prime \prime} \cos \varphi^{\prime \prime} \sin \varphi \\
& +\sin \theta^{\prime \prime} \sin \varphi^{\prime \prime} \cos \varphi\left[1+\delta(\varphi) \mu\left(\theta^{\prime \prime}\right)+\gamma(\varphi) \Delta(\varphi) \mu^{\prime}\left(\theta^{\prime \prime}\right)\right] \\
& -\cos \theta^{\prime \prime} \cos \varphi^{\prime \prime} \cos \varphi \gamma(\varphi) \\
\rho^{-1} X \cdot \underline{k}= & \sin \varphi^{\prime \prime} \sin \varphi-\cos \varphi^{\prime \prime} \cos \varphi\left[1+\delta(\varphi) \mu\left(\theta^{\prime \prime}\right)+\gamma(\varphi) \Delta(\varphi) \mu^{\prime}\left(\theta^{\prime \prime}\right)\right] .
\end{aligned}
$$

To show $X$ is never parallel to $\underline{i}$, we need to show $\rho^{-1} X \cdot \underline{j}$ and $\rho^{-1} X \cdot \underline{k}$ never vanish simultaneously.

Near the poles $X \cdot \underline{k}$ does not vanish. Near the equator for (a) $\theta^{\prime \prime}$ near $\pm \pi / 2, X \cdot \underline{k}$ does not vanish (where we pushed up), and for (b) $\theta^{\prime \prime}$ near $0, \pi$, $X \cdot \underline{j}$ does not vanish. We divide up the regions as follows:

(1) $-\beta \leqslant \varphi \leqslant \beta,-\pi / 3 \leqslant \theta^{\prime \prime} \leqslant \pi / 3$ or $2 \pi / 3 \leqslant \theta^{\prime \prime} \leqslant 4 \pi / 3$ (use $X \cdot \underline{j}$ ),

(2) $|\varphi| \geqslant \beta$ (use $X \cdot \underline{k}$ ),

(3) $\alpha \leqslant|\varphi| \leqslant \beta, \pi / 3 \leqslant \theta^{\prime \prime} \leqslant 2 \pi / 3$ or $4 \pi / 3 \leqslant \theta^{\prime \prime} \leqslant 5 \pi / 3$ (use $X \cdot \underline{k}$ ),

(4) $-\alpha \leqslant \varphi \leqslant \alpha, \pi / 3 \leqslant \theta^{\prime \prime} \leqslant 2 \pi / 3$ or $4 \pi / 3 \leqslant \theta^{\prime \prime} \leqslant 5 \pi / 3$ (use $X \cdot \underline{k}$ ), We separate cases (3) and (4) because different terms dominate. We make the following assumptions on $\gamma, \delta$, and $\Delta$ :

$|\gamma(\varphi)| \geqslant 50$ for $-\beta \leqslant \infty \leqslant \beta$,

$|\delta(\varphi)| \leqslant 1$,

$|\Delta(\varphi) \gamma(\varphi)| \leqslant 4$, 


$$
\begin{aligned}
& \Delta(\varphi) \gamma(\varphi)=-4 \text { for }-\alpha \leqslant \varphi \leqslant \alpha, \\
& \left|\mu\left(\theta^{\prime \prime}\right)\right|<1 / 2, \\
& \left|\mu^{\prime}\left(\theta^{\prime \prime}\right)\right|<1 .
\end{aligned}
$$

Remember $\mu\left(\theta^{\prime \prime}\right)=-1 / 2 \sin 2 \theta^{\prime \prime}$. We may need to make $\delta(\varphi)$ smaller and hence $\gamma(\varphi)$ larger in cases (3) and (4). Since $\Delta(\varphi)=0$ for $|\varphi| \geqslant \beta$, we have that $\left|\varphi^{\prime \prime}\right| \geqslant$ $\beta$ if and only if $|\varphi| \geqslant \beta\left(\varphi^{\prime \prime}=\varphi+\Delta \mu\right)$. Now for the cases.

Case (1). Assume $-\beta \leqslant \varphi, \varphi^{\prime \prime} \leqslant \beta$, and $-\pi / 3 \leqslant \theta^{\prime \prime} \leqslant \pi / 3$ or $2 \pi / 3 \leqslant \theta^{\prime \prime} \leqslant$ $4 \pi / 3$. Then

$$
\begin{aligned}
\sec \theta^{\prime \prime} \sec \varphi^{\prime \prime} \sec \varphi X \cdot \underline{j}= & -\gamma(\varphi)+\tan \theta^{\prime \prime} \tan \varphi \\
& +\tan \theta^{\prime \prime} \tan \varphi^{\prime \prime}\left[1+\delta(\varphi) \mu\left(\theta^{\prime \prime}\right)+\gamma(\varphi) \Delta(\varphi) \mu^{\prime}\left(\theta^{\prime \prime}\right)\right] \\
> & 50-(\sqrt{3}) 3+(\sqrt{3}) 3[1+1 / 2+4]>0 .
\end{aligned}
$$

Case (2). Assume $|\varphi|,\left|\varphi^{\prime \prime}\right| \geqslant \beta$. Then

$$
\sec \varphi^{\prime \prime} \sec \varphi X \cdot \underline{k}=\tan \varphi^{\prime \prime} \tan \varphi-\gamma(\varphi) \Delta(\varphi) \mu^{\prime}\left(\theta^{\prime \prime}\right)-1-\delta(\varphi) \mu\left(\theta^{\prime \prime}\right)
$$

$$
\geqslant 4-0-1-1 / 2>0 \text {. }
$$

Case (3). Assume $\alpha \leqslant|\varphi| \leqslant \beta$, and $\pi / 3 \leqslant \theta^{\prime \prime} \leqslant 2 \pi / 3$ or $4 \pi / 3 \leqslant \theta^{\prime \prime} \leqslant 5 \pi / 3$. By taking $\Delta(\varphi)$ smaller, we can insure that $\left|\tan \varphi^{\prime \prime}\right|>1$ so $\tan \varphi \tan \varphi^{\prime \prime}>2\left(\varphi^{\prime \prime}=\right.$ $\varphi+\Delta \mu)$. Then, since $-\gamma(\varphi) \Delta(\varphi) \mu^{\prime}\left(\theta^{\prime \prime}\right) \geqslant 0$,

$$
\begin{aligned}
\sec \varphi^{\prime \prime} \sec \varphi X \cdot \underline{k} & \geqslant \tan \varphi^{\prime \prime} \tan \varphi-1-\delta(\varphi) \mu\left(\theta^{\prime \prime}\right) \\
& \geqslant 2-1-1 / 2>0 .
\end{aligned}
$$

Case (4). Assume $|\varphi|<\alpha$, and $\pi / 3 \leqslant \theta^{\prime \prime}<2 \pi / 3$ or $4 \pi / 3<\theta^{\prime \prime}<5 \pi / 3$. For $\Delta(\varphi)$ small enough, we have $\tan \varphi^{\prime \prime} \tan \varphi>-1 / 4\left(\varphi^{\prime \prime}=\varphi+\Delta \mu\right)$, i.e. $\varphi$ and $\varphi^{\prime \prime}$ have the same sign except for small $\varphi$ and $\varphi^{\prime \prime}$. Also $-\gamma(\varphi) \Delta(\varphi) \mu^{\prime}\left(\theta^{\prime \prime}\right)=$ $-4 \cos 2 \theta^{\prime \prime} \geqslant 2$ for these ranges of $\theta^{\prime \prime}$. Therefore

$$
\begin{aligned}
\sec \varphi^{\prime \prime} \sec \varphi X \cdot \underline{k} & =\tan \varphi^{\prime \prime} \tan \varphi-\gamma(\varphi) \Delta(\varphi) \mu^{\prime}\left(\theta^{\prime \prime}\right)-1-\delta(\varphi) \mu\left(\theta^{\prime \prime}\right) \\
& \geqslant-1 / 4+2-1-1 / 2=1 / 4>0 .
\end{aligned}
$$

This completes the proof of Lemma 2 and hence the theorem.

3. Appendix: Construction of the $D A$ diffeomorphism. We begin with a hyperbolic toral automorphism $g: T^{n} \rightarrow T^{n}$ with the following properties: $g$ is induced by a linear automorphism $L: R^{n} \rightarrow R^{n}$ which can be represented by an integer matrix with determinant 1 , with one real eigenvalue $\lambda$ which satisfies $0<\lambda<1$ and all other eigenvalues greater than one in absolute value.

Choose a real valued $C^{\infty}$ function $\rho(t)$ with graph as in the figure below. 


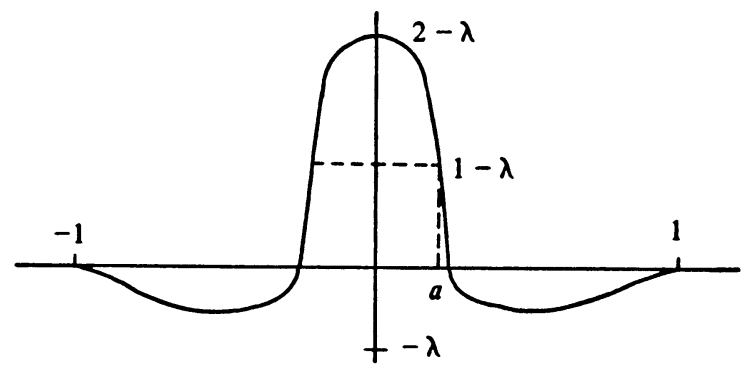

We summarize the properties we wish $\rho$ to have:

(1) $\rho(t)=\rho(-t)$,

(2) $\rho(t)=0$ if $|t| \geqslant 1, \rho(t)=2-\lambda$ on a neighborhood of 0 ,

(3) $\rho(t)>-\lambda$,

(4) $\int_{0}^{1} \rho(t) d t=0$,

(5) $\rho(a)=1-\lambda, \rho(t)<1-\lambda$ for $|t|>a$ and $\rho(t)>1-\lambda$ for $|t|<a$.

Now define $\psi: R \rightarrow R$ by $\psi(x)=\lambda x+\int_{0}^{x} \rho(t) d t$. Note that $\psi$ is a diffeomorphism, since $\psi^{\prime}(x)=\lambda+\rho(x)>0$ for all $x$, and that $\psi(x)=\lambda x$ for $|x|$ $\geqslant 1$. Also $\psi(x)=2 x$ for all $x$ in a neighborhood of 0 .

Since $\rho(t)>1-\lambda$ for $|t|<a$, there is a $b>a$ such that $\psi(t)>t$ for all $0<t \leqslant b$. Let $\bar{\lambda}=\sup _{|x|>b} \psi^{\prime}(x)$, then $\bar{\lambda}<1$, since $\rho(t)<1-\lambda$ when $|t|>$ a. We can now construct the $D A, f: T^{n} \rightarrow T^{n}$.

Let $\theta: R \rightarrow R$ be a $C^{\infty}$ bump function satisfying $0 \leqslant|\theta(t)| \leqslant 1, \theta(t) \equiv$ 1 on $[-b, b]$ and $\theta(t) \equiv 0$ for $|t| \geqslant 1$. Choose linear local coordinates $u_{1}, \ldots$, $u_{n}$ on a neighborhood $U$ of 0 in $T^{n}$, such that $u_{1}$ is parallel to the eigendirection corresponding to $\lambda$ and $u_{2}, \ldots, u_{n}$ span the complimentary expanding space. We suppose $U=\left\{g \mid \Sigma_{i=1}^{n} u_{i}^{2}(q)<4\right\}$. We now define $f$ by altering $g$ on $U$ as follows: Let $u_{i}(f(q))=u_{i}(g(q))$ for $2 \leqslant i \leqslant n$ and let $u_{1}(f(q))=\psi\left(u_{1}(q)\right) \theta(r)$ $+u_{1}(g(q))(1-\theta(r))$, where $r=\left(\sum_{i=2}^{n} u_{i}(q)^{2}\right)^{1 / 2}$. Note, since $\psi(x)=\lambda x$ for $|x| \geqslant 1, f$ agrees with $g$ outside $\left\{q \mid \sum_{i=1}^{n} u_{i}(q)^{2} \leqslant 1\right\}$. On a neighborhood of 0 in $T^{n}, f$ is an expanding map, linear in the $u_{i}$ coordinates $(\psi(x)=2 x$ for $x$ in a neighborhood of 0 ). It is not difficult to check that $f$ is a diffeomorphism on $T^{\boldsymbol{n}}$.

We note that $f$ leaves invariant the lines $u_{2}=$ const, $\ldots, u_{n}=$ const on $U$ so that in fact $f$ leaves invariant the foliation of stable manifolds of $g$ on all of $T^{n}$. Thus if $E^{s}$ is the stable bundle of $g$, this bundle is invariant under $T f$. The unstable bundle $E^{u}$ is not invariant under $T f$.

Now let $V=\left\{q \in U|| u_{1}(q) \mid<b\right.$ and $\left.\left(\sum_{i=2}^{n} u_{i}(q)^{2}\right)^{3 / 2}<b\right\}$. If $x \in U-$ $V$ and $v \in E_{x}^{s}$ then by construction $|T f(v)| \leqslant \bar{\lambda}|v|$ (recall $1>\bar{\lambda}>\lambda$ ). Hence we have this inequality on all of $T^{n}-V$ (since it holds with $\lambda$ instead of $\bar{\lambda}$ on $\left.T^{n}-U\right)$.

One checks easily that $f(V) \supset V$ and we wish now to show the hyperbolicity of $f$ on $\Lambda=\bigcap_{m>0} f^{m}\left(T^{n}-V\right)$, which is clearly a compact $f$-invariant set. 
To do this we consider $\Gamma=\Gamma^{s} \oplus \Gamma^{u}$ where $\Gamma^{s}=\left\{C^{0}\right.$ sections of $\left.E^{s}(g)\right\}$ and $\Gamma^{u}=\left\{C^{0}\right.$ sections of $\left.E^{u}(g)\right\}$ and define $f_{\#}(\gamma)=T f \circ \gamma \circ f^{-1}$ and $g_{\#}=T g \circ$ $\gamma \circ g^{-1}$ for $\gamma=\gamma^{s}+\gamma^{u} \in \Gamma$.

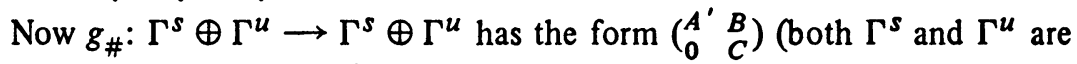
invariant under $\left.g_{\#}\right)$ where $\left|A^{\prime}\left(\gamma^{s}\right)\right| \leqslant \lambda\left|\gamma^{s}\right|$ and $\left|C^{n}\left(\gamma^{u}\right)\right| \geqslant K r^{n}\left|\gamma^{u}\right|$, for some $K>0$, and $r>1$. The map $f_{\#}: \Gamma^{s} \oplus \Gamma^{u} \rightarrow \Gamma^{s} \oplus \Gamma^{u}$ has the form $\left(\begin{array}{ll}A & B \\ 0 & C\end{array}\right)$ where $C: \Gamma \rightarrow \Gamma^{u}$ is the same as in the expression for $g$. To show that $\Lambda$ is a hyperbolic set it suffices to show $\left.f_{\#}\right|_{\Gamma_{\Lambda}}$ is hyperbolic where $\Gamma_{\Lambda}=\left\{\left.\gamma\right|_{\Lambda}: \gamma \in \Gamma\right\}$ (cf. Lemma 1 of [1]). Now $f_{\#}$ restricted to $\Gamma_{\Lambda}$ has the form $\left(\begin{array}{ll}A & B \\ 0 & C\end{array}\right)$ where $C: \Gamma_{\Lambda}^{u}$ $\rightarrow \Gamma_{\Lambda}^{u}$ is invertible and expanding since $\left|C^{n}\left(\gamma^{u}\right)\right| \geqslant K r^{n}\left|\gamma^{u}\right|$. Also $A: \Gamma_{\Lambda}^{s} \rightarrow$ $\Gamma_{\Lambda}^{s}$ satisfies $\left|A\left(\gamma^{s}\right)\right| \leqslant \bar{\lambda}\left|\gamma^{s}\right|$ since $\Lambda<T^{n}-V$ and $|T f(v)| \leqslant \bar{\lambda}|v|$ if $v \in E_{x}^{s}$, $x \in T^{n}-V$. Wc prove that if $\mu \in \mathrm{C}$ and $|\mu|=1$ then $f_{\#}-\mu I: \Gamma_{\Lambda} \rightarrow \Gamma_{\Lambda}$ is invertible by exhibiting an inverse, namely

$$
\left(\begin{array}{cc}
(A-\mu I)^{-1} & -(A-\mu I)^{-1} B(C-\mu I)^{-1} \\
0 & (C-\mu I)^{-1}
\end{array}\right) \text {. }
$$

Thus $\left.f_{\#}\right|_{\Gamma_{\Lambda}}$ is hyperbolic. It is clear that the stable manifolds of $\Lambda$ are leaves of the stable foliation of $g$ restricted to $T^{n}-\{0\}$.

REMARK. The above construction does not work if the stable dimension of the original linear map is greater than one. The difficulty is that the maximal invariant set does not have a constant splitting. What is happening is not well understood.

\section{REFERENCES}

1. J. Franks, Differentiably $\Omega$-stable diffeomorphisms, Topology 11 (1972), 107-113. MR $45 \# 6031$.

2. M. W. Hirsch, On invariant subsets of hyperbolic sets, Essays on Topology and Related Topics (Mèmoires dédiés á Georges de Rham), Springer, New York, 1970., pp. 126-135. MR 41 \#9275.

3. M. W. Hirsch and C. C. Pugh, Stable manifolds and hyperbolic sets, Proc. Sympos. Pure Math., vol. 14, Amer. Math. Soc., Providence, R.I., 1970, pp. 133-163. MR 42 \#6872.

4. Z. Nitecki, Differentiable dynamics, MIT Press, Cambridge, Mass., 1971.

5. R. Mañé, Expansive diffeomorphisms, Lecture Notes in Math., vol. 468, Springer-Verlag, Berlin and New York, 1975, pp. 162-174.

7. R. V. Sacker and G. R. Sell, Existence of dichotomies and invariant splittings for linear differential systems. I. J. Differential Equations 15 (1974), 429-458. MR 49 \#6209.

8. - Existence of dichotomies and invariant splittings for linear differential systems. II (to appear).

9. J. Selgrade, Isolated invariant sets for flows on vector bundles, Trans. Amer. Math. Soc. 203 (1975), 359-390.

10. S. Smale, Differentiable dynamical systems, Bull. Amer. Math. Soc. 73 (1967), 747-817. MR 37 \#3598; erratum, 39, p. 1593.

11. R. F. Williams, The "DA" maps of Smale and structural stability, Proc. Sympos. Pure Math., vol. 14, Amer. Math. Soc., Providence, R. I., 1970, pp. 329-334. MR 41 \#9296.

DEPARTMENT OF MATHEMATICS, NORTHWESTERN UNIVERSITY, EVANSTON, ILLINOIS 60201 